\title{
Evaluation of D-Dimer in Screening Deep Vein Thrombosis in Hospitalized Japanese Patients with Acute Medical Diseases/Episodes
}

\author{
Hiroshi Matsuo, MD, ${ }^{1}$ Yoshie Nakajima, MD, ${ }^{2}$ Tomohiro Ogawa, MD, PhD, ${ }^{3}$ Makoto Mo, MD, PhD, ${ }^{4}$ \\ Junichi Tazaki, MD, ${ }^{5}$ Takahiro Doi, MD, ${ }^{5}$ Norikazu Yamada, MD, PhD, ${ }^{6}$ Takeo Suzuki, BP, ${ }^{7}$ and \\ Hiromu Nakajima, MD, PhD7
}

Objective: To investigate the usefulness of D-dimer as a screening method as well as to explore potent predictors of deep vein thrombosis (DVT) in hospitalized Japanese patients with acute medical diseases/episodes.

Methods and Subjects: This study was a multi-center, prospective, observational study. The inclusion criteria were hospitalized patients at high risk of developing venous thromboembolism with; (1) congestive heart failure, acute exacerbation of chronic obstructive pulmonary disease, infectious diseases, or inflammatory diseases, (2) bed rest $\geq 4$ days, and (3) $\geq 60$ years old. D-dimer was measured on the same day as ultrasonography. Multivariate logistic regression analysis was performed to investigate predictors associated with the presence of DVT.

Results: Sixty-nine patients were enrolled. The prevalence of DVT was 33.3\% (23/69; 95\% C.I., $19.4 \%$ to $47.3 \%)$. D-dimer was measured in 42 patients and the sensitivity and negative predictive value reached $100 \%$, while the specificity (13.3\%) and positive predictive value (31.6\%) were low (cut-off value: 0.9 or $1.0 \mu \mathrm{g} / \mathrm{mL}$ ). Statistically significant predictor was not assigned.

\footnotetext{
${ }^{1}$ Matsuo Vascular Ultrasound Laboratory, Matsuo Clinic, Suita, Osaka, Japan

${ }^{2}$ Department of Cardiology, Saitama Medical University International Medical Center, Hidaka, Saitama, Japan ${ }^{3}$ Cardiovascular Disease Center, Fukushima Daiichi Hospital, Fukushima, Fukushima, Japan

${ }^{4}$ Department of Cardiovascular Surgery, Yokohama Minami Kyousai Hospital, Yokohama, Kanagawa, Japan

${ }^{5}$ Department of Cardiovascular Medicine, Graduate School of Medicine, Kyoto University, Kyoto, Kyoto, Japan

${ }^{6}$ Department of Cardiology and Nephrology, Mie University Graduate School of Medicine, Tsu, Mie, Japan

${ }^{7}$ Development \& Medical Affairs Division, GlaxoSmithKline K.K., Shibuya, Tokyo, Japan
}

Received: March 28, 2016; Accepted: June 22, 2016 Corresponding author: Hiroshi Matsuo, MD. Matsuo Vascular Ultrasound Laboratory, Matsuo Clinic, 16-9 Enoki-Cho, Suita Osaka, 564-0053, Japan

Tel: +8166388 0041, Fax: +81663880166

E-mail: hiro-matu@fan.hi-ho.ne.jp
Conclusion: As the sensitivity and negative predictive value of D-dimer reached $100 \%$, D-dimer have a role in excluding patients who might otherwise undergo diagnostic imaging for DVT in hospitalized Japanese patients with acute medical diseases/episodes.

Keywords: deep vein thrombosis, Japanese patients, medical
patients, D-dimer, predictors

\section{Introduction}

D-dimer has been widely utilized for the screening and diagnosis of venous thromboembolism (VTE) ${ }^{1,2)}$ and was included in the diagnostic algorithm of "Guidelines for the Diagnosis, Treatment and Prevention of Pulmonary Thromboembolism and Deep Vein Thrombosis."3) While some reports have been published in Japan, most of which refer only to surgical and not to non-surgical patients. The subjects of these reports were postoperative orthopedic patients, ${ }^{4,5)}$ postoperative patients with gynecological cancers, ${ }^{6)}$ ovarian cancer or borderline malignancy, ${ }^{7)}$ stroke patients, ${ }^{8)}$ and orthopedic patients or those with other forms of cancer, cardiovascular disease, or gastrointestinal disease. $^{9-12)}$ Most of the subjects in retrospective study were symptomatic patients, prospective study was required in order to clarify the actual situation including asymptomatic patients.

Although the risk factors for DVT in medical practice are described in the "Japanese Guideline for Prevention of Venous Thromboembolism" (prevention guideline), ${ }^{13)}$ reports from Japan are limited to patients with heart failure, ${ }^{14,15)}$ stroke, ${ }^{8)}$ acute intracerebral hemorrhage, ${ }^{16)}$ and severe motor and intellectual disabilities. ${ }^{17,18)}$ The increased D-dimer levels are known to associate with aging and other medical/pathological conditions other than thrombosis. ${ }^{19)}$ Therefore, in the hospitalized elderly patients with acute medical diseases/episodes and these conditions, specificity and positive predictive value were expected to be low compared to 
the patients without these conditions. High sensitivity and negative predictive value are required crucially as screening test, and at the same time showing its specificity and positive predictive value should be associated so that we can employ age-adjusted high cut-off value for elderly patients.

We enrolled non-surgical Japanese patients judged to be at high risk of developing VTE as defined by the prevention guideline, ${ }^{13}$ ) and evaluated the usefulness of D-dimer in screening for DVT. We also explored potent predictors for the presence of DVT among the known risk factors.

\section{Materials and Methods}

Prior to start the study, the protocol formulated by investigators was reviewed and approved by the institutional review board/ ethical committee of each medical site. The study was performed in accordance to the Declaration of Helsinki version 2008. The investigator explained the purpose and methods of the study to each subject before obtaining written informed consent.

Hospitalized patients identified as high risk of developing VTE were enrolled in the study. Risk factors considered were those referred to in the prevention guideline. ${ }^{13)}$ Inclusion criteria were patients with; (a) acute exacerbation of congestive heart failure (CHF) in Class III or IV of NYHA (New York Heart Association) functional classification, acute exacerbation of chronic obstructive pulmonary disease (COPD), infectious diseases, or inflammatory diseases (including rheumatological diseases), (b) expected to require bed rest for at least four days, and (c) age of at least 60 years. Patients were excluded if they had received treatment for VTE, undergone surgery or been injured within the 3 months prior to bed rest, a diagnosis of VTE at hospitalization, or were receiving treatment with anticoagulants. As well as patients who had complete bed rest, we included patients where there was a temporary suspension of bed rest (e.g., in order to go to the toilet) during the bed rest period. We also counted rest period in another site or home just before hospitalization in study site.

After receiving informed consent, the subject was assessed for the presence of DVT by lower extremity ultrasonography (US) as well as undergoing a physical examination and laboratory tests. The following information was obtained: demographic variables, lifestyle variables, and comorbidities (see Table 1). Laboratory tests for thrombophilia were performed for subjects with DVT. If the rest period was less than four days contrary to expectations, investigation was discontinued.

D-dimer was measured on the same day that each patient underwent US. The assays were performed in the laboratory of each study site using the same test cut-off levels that the study site had routinely used ( 0.9 or $1.0 \mu \mathrm{g} / \mathrm{mL})$. The assay methods included latex agglutination and latex photometric immunoassay.

\section{Evaluation of DVT}

Diagnosis by US is non-invasive, and was standardized in the 2008 guideline "Criteria for ultrasound diagnosis of deep venous thrombosis of lower extremities" by the Japan Society of Ultrasonics in Medicine. ${ }^{20)}$ We thus considered that diagnosis using US was reproducible and reliable. A US was performed between Day 6 of bed rest and Day 14 of hospitalization in study site. Whenever DVT was suspected from symptoms, we immediately conducted US to assess the patient. If bed rest was cancelled before more than 2 days of US planned date, US was performed on the day or the next day of bed rest cancel. In this study, the US targeted the following lower extremity veins from the iliac crest to the lower leg: common iliac vein, external iliac vein, internal iliac vein, common femoral vein, superficial femoral vein, deep femoral vein, popliteal vein, gastrocnemius vein, peroneal vein, posterior tibial vein, anterior tibial vein, and soleus vein. When the thrombus was observed or suspected in illiac or femoral veins, we also tested inferior vena cava to confirm proximal end. The test was performed in the supine position at the pelvic and femoral regions, and in the sitting position at the popliteal and crural regions. However, if the sitting position was difficult due to the patient's condition, the test in supine position was also allowed. When testing the pelvic region, a 3.5 $\mathrm{MHz}$ convex probe or sector probe was used. For testing the femoral, popliteal, and crural regions, a 7 to $10 \mathrm{MHz}$ linear probe or a 3.5 to $7.5 \mathrm{MHz}$ convex probe was used. Compression sonography with B-mode was adopted for morphological diagnosis. For evaluation of blood flow, Color Doppler or pulse Doppler methods were also employed. Distinction of new or old thrombus was also performed based on the following information: stenosis and distention of vein, floating, regression, consistency, surface character, brightness, and homogeneity of thrombus, perfusion defect, recanalization in thrombus, and collateral in branch. We defined as proximal DVT when the proximal end was observed in the following veins: inferior vena cava, common iliac vein, external iliac vein, internal iliac vein, common femoral vein, superficial femoral vein, deep femoral vein, or popliteal vein. The detection of pulmonary thromboembolism was not a part of this study. Prior to the start of the study, a workshop was held in which the investigators and the medical technologists were given a detailed explanation and thorough training on the US procedure.

We established an independent central evaluation committee to minimize differences in evaluation among the study sites. A physician (Hiroshi Matsuo) who was an 
Table 1 Characteristics of hospitalized patients with congestive heart failure, chronic obstructive pulmonary disease, infectious diseases, or inflammatory diseases

\begin{tabular}{|c|c|}
\hline Variables & $\mathrm{N}=69$ \\
\hline Age* (years) & $80.5 \pm 9.5$ \\
\hline Women & $36(52.2 \%)$ \\
\hline $\mathrm{BMI}\left(\mathrm{kg} / \mathrm{m}^{2}\right)$ & $21.6 \pm 5.7$ \\
\hline Systolic blood pressure (mmHg) & $125.2 \pm 20.7$ \\
\hline Diastolic blood pressure (mmHg) & $69.1 \pm 16.7$ \\
\hline Duration of rest* (days) & $9.8 \pm 4.8$ \\
\hline Smoking history (Male) (current or past) & $16(48.5 \%)$ \\
\hline Smoking history (Female) (current or past) & $3(8.3 \%)$ \\
\hline Habitual drinker (Male) (current and past) & $16(48.5 \%)$ \\
\hline Habitual drinker (Female) (current and past) & $2(5.6 \%)$ \\
\hline Recent regular exercise (once or more in one week) & $14(20.3 \%)$ \\
\hline Catheterization within last three months ${ }^{\dagger}$ & $13(18.8 \%)$ \\
\hline \multicolumn{2}{|l|}{ Reason for hospitalization } \\
\hline Congestive heart failure & $44(63.8 \%)$ \\
\hline Infectious diseases & $14(20.3 \%)$ \\
\hline Pneumonia & $9(13.0 \%)$ \\
\hline Bronchopneumonia & $3(4.3 \%)$ \\
\hline Aspiration pneumonia & $1(1.4 \%)$ \\
\hline Cholangitis & $1(1.4 \%)$ \\
\hline Acute exacerbation of COPD & $9(13.0 \%)$ \\
\hline Inflammatory diseases & $2(2.9 \%)$ \\
\hline Articular rheumatism with pulmonary fibrosis & $1(1.4 \%)$ \\
\hline Nephrotic syndrome & $1(1.4 \%)$ \\
\hline Mechanical prophylaxis & $8(11.6 \%)$ \\
\hline Taking antiplatelet agents & $17(24.6 \%)$ \\
\hline \multicolumn{2}{|l|}{ Comorbidities } \\
\hline Malignancy & $13(18.8 \%)$ \\
\hline Diabetes & $13(18.8 \%)$ \\
\hline Dyslipidemia & $13(18.8 \%)$ \\
\hline Hyperuricemia & $22(31.9 \%)$ \\
\hline Paralysis & $6(8.7 \%)$ \\
\hline Hypertension & $46(66.7 \%)$ \\
\hline Angina pectoris & $17(24.6 \%)$ \\
\hline Myocardial infarction & $7(10.1 \%)$ \\
\hline Thrombophilia & $1(1.4 \%)$ \\
\hline Arrhythmia & $27(39.1 \%)$ \\
\hline Stroke & $16(23.2 \%)$ \\
\hline Aortic aneurysm & $4(5.8 \%)$ \\
\hline COPD & $15(21.7 \%)$ \\
\hline Asthma & $6(8.7 \%)$ \\
\hline Gastroduodenal ulcer & $5(7.2 \%)$ \\
\hline Chronic hepatitis & $1(1.4 \%)$ \\
\hline Hepatic cirrhosis & $1(1.4 \%)$ \\
\hline Nephrosis syndrome & $3(4.3 \%)$ \\
\hline
\end{tabular}

"Age and duration of rest are values at the time of US and other data are values at the registration. ${ }^{\dagger}$ Catheterization was investigated regardless purpose and site. Values are expressed as mean \pm standard deviation for continuous variables. Italics indicate breakdown of the disease. BMI: body mass index; VTE: venous thromboembolism; COPD: chronic obstructive pulmonary disease

author of the guideline "Criteria for ultrasound diagnosis of deep venous thrombosis of lower extremities" ${ }^{20)}$ was assigned to be a central evaluation committee member. Medical site where the central evaluation committee member belonged did not register the subjects to stand as an independent manner. Investigators sent all moving image, following still images (both common iliac veins confluence section, right/left common femoral veins, right/left popliteal veins, and right/left crural veins including soleus veins) and reports on the US of the lower extremity vein to the independent central evaluation committee. If there were questions on the images or reports, the central evaluation committee contacted investigators or medical technologists. Using a standardized approach, the committee evaluated and determined presence of DVT based on these images and information. The US results in this study were based on the final committee evaluations. 
Table 2 Characteristics of patients classified by the presence or absence of DVT

\begin{tabular}{|c|c|c|c|}
\hline & DVT $(+)(n=23)$ & DVT $(-)(n=46)$ & $P$ value $\ddagger$ \\
\hline Women & $13(56.5 \%)$ & $23(50.0 \%)$ & 0.799 \\
\hline Age* (years) & $79.5 \pm 10.3^{\dagger}$ & $81.0 \pm 9.2$ & 0.525 \\
\hline BMI $\left(\mathrm{kg} / \mathrm{m}^{2}\right)$ & $20.7 \pm 5.6$ & $22.0 \pm 5.7$ & 0.399 \\
\hline Systolic blood pressure (mmHg) & $124.6 \pm 22.6$ & $125.5 \pm 19.9$ & 0.861 \\
\hline Diastolic blood pressure $(\mathrm{mmHg})$ & $67.3 \pm 11.2$ & $70.1 \pm 18.9$ & 0.487 \\
\hline Duration of rest* (days) & $11.0 \pm 6.9$ & $9.2 \pm 3.3$ & 0.376 \\
\hline Smoking history (Male) & $6(26.1 \%)$ & $10(21.7 \%)$ & 0.465 \\
\hline Smoking history (Female) & $1(4.3 \%)$ & $2(4.3 \%)$ & 1.000 \\
\hline Habitual drinker (Male) & $6(26.1 \%)$ & $10(21.7 \%)$ & 0.465 \\
\hline Habitual drinker (Female) & $1(4.3 \%)$ & $1(2.2 \%)$ & 1.000 \\
\hline Recent regular exercise (once or more in one week) & $5(21.7 \%)$ & $9(19.6 \%)$ & 1.000 \\
\hline Catheterization within last three months ${ }^{\dagger}$ & $5(21.7 \%)$ & $8(17.4 \%)$ & 0.743 \\
\hline \multicolumn{4}{|l|}{ Comorbidities } \\
\hline Malignancy & $6(26.1 \%)$ & $7(15.2 \%)$ & 0.334 \\
\hline Chemotherapy for malignancy & $3(13.0 \%)$ & $3(6.5 \%)$ & 1.000 \\
\hline Diabetes & $3(13.0 \%)$ & $10(21.7 \%)$ & 0.520 \\
\hline Dyslipidemia & $2(8.7 \%)$ & $11(23.9 \%)$ & 0.194 \\
\hline Hyperuricemia & $7(30.4 \%)$ & $15(32.6 \%)$ & 1.000 \\
\hline Paralysis & $0(0.0 \%)$ & $6(13.0 \%)$ & 0.168 \\
\hline Hypertension & $14(60.9 \%)$ & $32(69.6 \%)$ & 0.589 \\
\hline Angina pectoris & $4(17.4 \%)$ & $13(28.3 \%)$ & 0.387 \\
\hline Myocardial infarction & $4(17.4 \%)$ & $3(6.5 \%)$ & 0.211 \\
\hline Thrombophilia & $1(4.3 \%)$ & $0(0.0 \%)$ & 0.333 \\
\hline Arrhythmia & $6(26.1 \%)$ & $21(45.7 \%)$ & 0.190 \\
\hline Heart failure & $16(69.6 \%)$ & $33(71.7 \%)$ & 1.000 \\
\hline Stroke & $3(13.0 \%)$ & $13(28.3 \%)$ & 0.229 \\
\hline Aortic aneurysm & $2(8.7 \%)$ & $2(4.3 \%)$ & 0.596 \\
\hline Chronic obstructive pulmonary disease & $8(34.8 \%)$ & $7(15.2 \%)$ & 0.119 \\
\hline Asthma & $0(0.0 \%)$ & $6(13.0 \%)$ & 0.168 \\
\hline Gastroduodenal ulcer & $0(0.0 \%)$ & $5(10.9 \%)$ & 0.161 \\
\hline Chronic hepatitis & $0(0.0 \%)$ & $1(2.2 \%)$ & 1.000 \\
\hline Hepatic cirrhosis & $0(0.0 \%)$ & $1(2.2 \%)$ & 1.000 \\
\hline Nephrosis syndrome & $1(4.3 \%)$ & $2(4.3 \%)$ & 1.000 \\
\hline
\end{tabular}

‡Statistical test methods: Fisher's exact test for categorical data, Student's t-test or Wilcoxon rank sum test for continuous data. "Age and duration of rest are values at the time of US and other data are values at the registration. ${ }^{+}$Catheterization was investigated regardless purpose and site. Values are expressed as mean \pm standard deviation for continuous variables. DVT: deep vein thrombosis; BMI: body mass index

\section{Data Analysis}

The frequency of DVT was calculated. The prevalence of potential risk factors for DVT (obesity, bed rest, smoking, diabetes, etc.) was compared between DVT-positive and -negative groups by Fisher's exact test, Student's t-test or Wilcoxon rank sum test.

The D-dimer levels were compared in relation to DVT present/absent and then the sensitivity, specificity, positive predictive value, and negative predictive value were calculated. The D-dimer data that were obtained on the same day as the US for each patient were included in the data analysis.

Continuous variables were summarized using descriptive statistics (mean \pm standard deviation $[\mathrm{SD}]$ ) and discrete variables were summarized using frequencies and percentage. The results of statistical tests were indicated by $P$ values with a significance level of $\alpha=0.05$. Logistic regression analysis was performed to investigate predictors associated with the presence of DVT. We chose following six potential predictors as independent variables: age, duration of rest, sex, taking antiplatelet agent, concomitant malignancy, and concomitant COPD. Statistical analyses were performed using R version 2.15.2 (R Foundation for Statistical Computing, Vienna, Austria).

\section{Results}

The study was conducted from September 2009 to August 2010 at six sites (Fukushima Daiichi Hospital, Saitama Medical University International Medical Center, Yokohama Minami Kyousai Hospital, Mie University Hospital, Kyoto University Hospital, and Takarazuka Municipal Hospital).

A total of 69 subjects ( 33 males and 36 females; mean age of $80.5 \pm 9.5$ years) were enrolled in the study. Forty-four patients had CHF, 14 had infectious diseases (nine with 
Table 3 Sensitivity, specificity, positive predictive value, and negative predictive value of D-dimer compared with the results of US

\begin{tabular}{lccc}
\hline \multicolumn{4}{c}{ US } \\
\hline D-dimer & DVT $(-)$ & DVT $(+)$ & Total \\
\hline High & 26 & 12 & 38 \\
Normal & 4 & 0 & 4 \\
Total & 30 & 12 & 42 \\
\hline
\end{tabular}

Sensitivity: $100 \%$, Specificity: $13.3 \%$, Positive predictive value: $31.6 \%$, Negative predictive value: $100 \%$. US: ultrasonography; DVT: deep vein thrombosis

pneumonia, three with bronchopneumonia, one with aspiration pneumonia and one with cholangitis), nine had COPD and two had inflammatory disease (one case of articular rheumatism with pulmonary fibrosis and one of nephrotic syndrome). No patients had histories of VTE and eight patients received mechanical prophylaxis (Table 1 ).

Details of subject characteristics by the presence or absence of DVT were shown in Table 2. No statistically significant difference was observed between DVT-positive $(n=23)$ and -negative $(n=46)$ groups in terms of baseline characteristics.

The calculated total prevalence of DVT was $33.3 \%$ (23/69) in patients with CHF, COPD, infectious diseases, or inflammatory diseases $(95 \%$ confidence interval: $19.4 \%-47.3 \%)$. Six subjects had proximal DVT and their proximal ends were common femoral vein in one subject, superficial femoral vein in two subjects, and popliteal vein in three subjects. Seventeen subjects had distal DVT and all of them had thrombi in soleus veins. Seventeen subjects had new thrombi and six had old thrombi only. Twenty out of 23 subjects $(87.0 \%)$ were asymptomatic. After re-evaluation by central evaluation committee, two cases were changed from DVT positive to negative, and four cases were changed from DVT negative to positive. The evaluations by site investigator and evaluation committee were the same for other 63 cases (19 DVT positive and 44 DVT negative cases).

The sensitivity, specificity, positive predictive value, and negative predictive value of $\mathrm{D}$-dimer compared with the results of US were summarized in a contingency table (Table 3). Forty-two patients had their D-dimer measured on the same day as they underwent US. The cut-off level adopted for the $\mathrm{D}$-dimer test was the same value (1.0 or 0.9 $\mu \mathrm{g} / \mathrm{mL}$ ) as that routinely used in each laboratory. A total of 38 patients were classified as high (i.e. abnormal) level including all 12 DVT positive patients. In contrast, four patients were classified as normal level while there were 30 DVT negative patients. These results showed that the sensitivity and negative predictive value reached $100 \%$, while the specificity $(13.3 \%)$ and positive predictive value

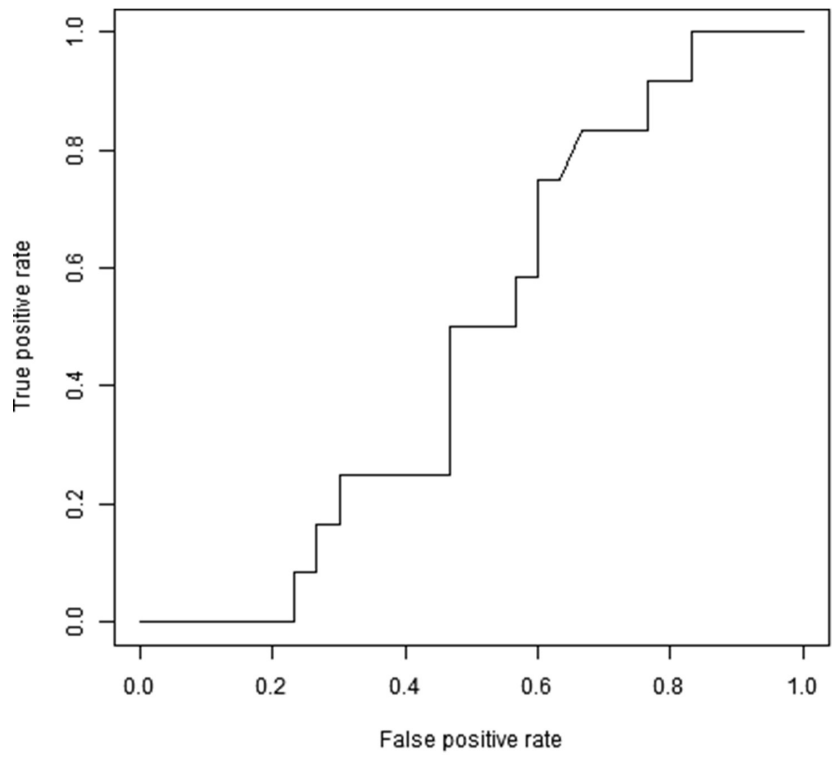

Fig. 1 Receiver Operating Characteristics (ROC) curve of D-dimer. When the cut-off level was $3.5 \mu \mathrm{g} / \mathrm{mL}$, the ROC curve was closest to the upper left corner. Sensitivity was $75 \%$ and specificity was $40 \%$ at that point.

(31.6\%) were low. We drew a Receiver Operating Characteristics (ROC) curve and examined the cut-off level (Fig. 1). When the cut-off level was $3.5 \mu \mathrm{g} / \mathrm{mL}$, the ROC curve was closest to the upper left corner. The sensitivity was $75 \%$, specificity was $40 \%$, the positive predictive value was $33.3 \%$, and the negative predictive value was $80 \%$ at that point.

The results of logistic regression analysis were shown in Table 4. No statistically significant predictor was found. $\mathrm{P}$ value of concomitant COPD was less than 0.1.

\section{Discussion}

Based on the lower extremity vein US, $33.3 \%$ (23/69; $95 \%$ CI, $19.4 \%$ to $47.3 \%$ ) of non-surgical patients with CHF, COPD, infectious diseases, or inflammatory diseases had DVT. The results of our study indicate that high risk patients exist in the non-surgical patient group with a frequency comparable to that of post-operative patients $(27.4 \%$ to $50.0 \%) .{ }^{21)}$ Notably, 20 patients (20/23: 87.0\%) with DVT were asymptomatic. In 2007 and later, new anticoagulants (fondaparinux sodium, enoxaparin sodium, edoxaban tosilate hydrate, rivaroxaban, and apixaban) became available in Japan, but VTE prevention is only indicated for patients undergoing surgery. Unfractionated heparin was approved also for non-surgical patients, it is necessary to manage the bleeding risk when using anticoagulants. For acutely ill hospitalized medical patients who were bleeding or at high risk for bleeding, American College 
Table 4 Logistic regression analysis

\begin{tabular}{lcccc}
\hline Variable & $\begin{array}{c}\text { Crude odds ratio } \\
(95 \% \mathrm{Cl})\end{array}$ & P value & $\begin{array}{c}\text { Adjusted odds ratio } \\
(95 \% \mathrm{Cl})\end{array}$ & P value \\
\hline Age (for 1 year) & $0.98(0.93-1.04)$ & 0.519 & $0.96(0.90-1.02)$ & 0.193 \\
Rest period (for 1 day) & $1.09(0.96-1.22)$ & 0.179 & $1.06(0.92-1.23)$ & 0.426 \\
Sex (women) & $1.30(0.47-3.56)$ & 0.610 & $1.89(0.60-5.95)$ & 0.277 \\
Taking antiplatelet agent & $0.34(0.09-1.34)$ & 0.125 & $0.32(0.07-1.37)$ & 0.124 \\
Comorbiditiy: malignancy & $1.97(0.57-6.73)$ & 0.281 & $1.82(0.42-7.91)$ & 0.423 \\
Comorbiditiy: COPD & $2.97(0.92-9.63)$ & 0.070 & $3.17(0.84-11.89)$ & 0.088 \\
\hline
\end{tabular}

$\mathrm{Cl}$ : confidence interval; COPD: chronic obstructive pulmonary disease

of Chest Physicians (ACCP) evidence-based clinical practice guideline recommended against anticoagulant thromboprophylaxis. ${ }^{22)}$ Since the new anticoagulants does not have indication of prevention for non-surgical patients and $87 \%$ of patients with DVT were asymptomatic in this study, asymptomatic VTE is more likely to be missed in non-surgical patients. However, due to limitations on examination time and cost, diagnostic imaging of the lower extremity vein cannot be performed for all the patients at high risk. D-dimer is useful as a screening test as it can narrow down the number of patients who need to undergo diagnostic imaging by excluding patients with a low D-dimer level. The results indicated that the sensitivity and negative predictive value of D-dimer reached $100 \%$, while the specificity $(13.3 \%)$ and positive predictive value $(31.6 \%)$ were low. In previous study for patients with acute stroke, high sensitivity $(89 \%)$ and specificity $(82 \%)$ were reported when a cut-off value was set as $5.5 \mu \mathrm{g} / \mathrm{mL} .^{8)}$ In that study, it was suggested that degree of D-dimer increase was not as much as the patients with acute stroke. Yamada et al. investigated DVT occurrence in non-surgical patients with various diseases including cerebrovascular diseases and heart diseases. DVT occurrence was $9.3 \%$ in patients with ischemic heart disease and $11.1 \%$ in patients with other heart diseases. ${ }^{23)}$ DVT occurrence in patients with heart diseases was not as high as occurrence in patients with cerebrovascular diseases $(25.7 \%)$. If we set higher cutoff value to increase specificity, sensitivity would be decreased. In this study, we employed cut-off value ( 0.9 or $1.0 \mu \mathrm{g} / \mathrm{mL}$ ) which was set by the laboratory of each site. In consideration that the purpose is screening, we think that it is better to focus on sensitivity even have low specificity and positive predictive value. Other than thrombosis, the following conditions have all been shown to be associated with increased D-dimer levels: aging, smoking, malignancy, infection, chronic inflammatory diseases, acute coronary syndromes, stroke, and congestive heart failure. ${ }^{19)}$ Therefore, there is a limitation to represent the definitive diagnosis of having DVT by testing D-dimer levels. However, the measurement of D-dimer levels may be useful as it can narrow down the number of patients that need to be examined by diagnostic imaging when it is used as a screening test prior to diagnostic imaging. We considered that many subjects even without thrombi or with old thrombi only of this study had factors that increase D-dimer level that resulted in low positive predictive value and specificity. We showed that the prevalence of DVT was fairly high even in non-surgical asymptomatic hospitalized patients with acute medical diseases/episodes and evaluation for DVT should be mandatory in such high risk patients. ${ }^{3)}$

We measured D-dimer only once in each institution and did not perform central measurement. The diagnostic accuracy of D-dimer observed in this study was not as high as that achieved in previous studies of surgical patients. ${ }^{4,5)}$ Because the condition of non-surgical patients before and after hospitalization varies substantially among individuals in comparison with patients undergoing elective surgery, we have to assume that there was a large variance in the timing of thrombus generation. Because D-dimer levels change depending on the blood collection time, ${ }^{4-6)}$ further study is necessary to evaluate the optimal blood collection time in non-surgical patients. Watanabe et al. reported that based on multiple logistic regression analysis, D-dimer at postoperative Day 4 was an independent marker of DVT but had no statistical significance on postoperative Day $1 .{ }^{4)}$ Kodama et al. reported that the D-dimer value gradually increased, peaked on postoperative Days 7 to 10, and then decreased. $\left.{ }^{6}\right)$

No statistically significant predictor was identified among the predefined risk factors by multivariate logistic regression analysis. Age, rest period, concomitant malignancy, and concomitant COPD were assigned as independent variables with reference to the risks specified in the prevention guidelines. ${ }^{13)}$ Sex was assigned based on a patient survey conducted by the Ministry of Health, Labour and Welfare. ${ }^{24)}$ Taking antiplatelet agent was assigned based on a review article by Watson et al. ${ }^{25)}$

A major strength of our study is that we investigated DVT prospectively even for asymptomatic patients. Since the 20 patients were asymptomatic among 23 patients with DVT, the findings could not be obtained from retrospective studies became clear. Most of the subjects in retrospective study are symptomatic patients. We also consider that multicenter study have a higher value more than a single-center study for generalizability. 
This study has potentially several limitations. Firstly, patients with asymptomatic pulmonary thromboembolism (PE) might be overlooked because the test for asymptomatic PE was not a part of this study. Secondly, regarding to investigate distal DVT by US in multicenter study, variance on test conditions, procedures of the medical technologist, and diagnosis remain concerned. To ensure the objectivity of the results for distal DVT by US, we standardized US procedures and established a central evaluation committee. For diagnostic accuracy of US compared with venography in lower extremity DVT, Urayama et al. reported that sensitivity of $86.8 \%$ and specificity of $100 \%$, and Pedersen et al. reported that sensitivity of $89 \%$ and specificity of $97 \% .{ }^{26,27)}$ It would be beneficial to evaluate the properties and analyze the characteristics of the thrombosis for predicting the risk of recurrence by US. ${ }^{28)}$ ACCP evidence-based clinical practice guideline recommended that additional other test was not performed when the thrombus was observed (regardless proximal or distal veins) or not by US. ${ }^{29)}$ Ultrasonography was performed in $87.7 \%$ and venography was performed in $2.8 \%$ of patients in a survey by Japanese Society of Phlebology in 2009. ${ }^{30)}$ Thirdly, the number of subjects in this study was small compared to the previous studies. Depending on the strength of relationship between predictors and outcomes, more subjects was likely needed in order to show statistical significance.

More than 5 years have passed since the end of this study. Although ACCP evidence-based clinical practice guidelines were revised during this period, there was not so much significant change regarding recommended prevention of VTE in non-surgical patients. Indications of new anticoagulants for VTE prevention are currently limited to surgical patients in Japan. And therefore we considered no significant change has occurred for VTE management in Japanese non-surgical patients, compared with this study execution period.

\section{Conclusion}

Based on the results of the lower extremity vein US, 33.3\% (23/69; $95 \%$ CI, $19.4 \%$ to $47.3 \%)$ of subjects were identified as having DVTs even among non-surgical patients with CHF, COPD, infectious diseases, or inflammatory diseases. D-dimer assay is considered to be useful as a screening test for DVT in hospitalized patients with acute medical diseases/episodes.

\section{Acknowledgments}

We would like to express our gratitude to all of the patients who participated in the study, participating investigators (Dr. Makoto Matsumura and Dr. Takashi Koide), medical technologists (Tetsuya Yamamoto, Kenji Kamata,
Masakazu Abe, Orie Kaneko, Hiroshi Sato, Tomoya Yoneda, Noriyuki Shinoda, Masumi Matsuda, Shinobu Fujii, and Nobuaki Asaoka) and other staff members who contributed to the study. Some of the findings have already been presented at The 31th Annual Meeting of Japanese Society of Phlebology.

\section{Disclosure Statement}

This study was sponsored by GlaxoSmithKline K.K. and the study costs were covered by their contract research fund. Hiroshi Matsuo was an external medical advisor and received compensation from GlaxoSmithKline K.K. for the advice. Yoshie Nakajima, Tomohiro Ogawa, Makoto Mo, Junichi Tazaki, Takahiro Doi, and Norikazu Yamada were contracted investigators for this study. M Mo received research fund from Daiichi Sankyo Company, Limited and Pfizer Japan Inc. outside the submitted work. N Yamada received lecture fee from Daiichi Sankyo Company, Limited outside the submitted work. Takeo Suzuki and Hiromu Nakajima are employees and Hiromu Nakajima is a stockholder of GlaxoSmithKline K.K.

\section{Author Contributions}

Study conception and design: HM, TS, HN; Data collection: YN, TO, MM, JT, TD, NY; Data analysis and interpretation: HM, TS, HN; Writing: TS; Critical review and revision: all authors; Final approval of the article: all authors; Accountability for all aspects of the work: all authors.

\section{References}

1) Stein PD, Hull RD, Patel KC, et al. D-dimer for the exclusion of acute venous thrombosis and pulmonary embolism: a systematic review. Ann Intern Med 2004; 140: 589-602.

2) Bozic M, Blinc A, Stegnar M. D-dimer, other markers of haemostasis activation and soluble adhesion molecules in patients with different clinical probabilities of deep vein thrombosis. Thromb Res 2002; 108: 107-14.

3) JCS Joint Working Group. Guidelines for the diagnosis, treatment and prevention of pulmonary thromboembolism and deep vein thrombosis (JCS 2009). Circ J 2011; 75: $1258-81$.

4) Watanabe H, Madoiwa S, Sekiya H, et al. Predictive blood coagulation markers for early diagnosis of venous thromboembolism after total knee joint replacement. Thromb Res 2011; 128: e137-43.

5) Niimi R, Hasegawa M, Sudo A, et al. Evaluation of soluble fibrin and D-dimer in the diagnosis of postoperative deep vein thrombosis. Biomarkers 2010; 15: 149-57.

6) Kodama J, Seki N, Masahiro S, et al. D-dimer level as a risk factor for postoperative venous thromboembolism in Japanese women with gynecologic cancer. Ann Oncol 2010; 21: 1651-6. 
7) Kawaguchi R, Furukawa N, Kobayashi H. Cut-off value of D-dimer for prediction of deep venous thrombosis before treatment in ovarian cancer. J Gynecol Oncol 2012; 23: 98-102.

8) Kuwashiro T, Toyoda K, Oyama N, et al. High plasma $\mathrm{D}$-dimer is a marker of deep vein thrombosis in acute stroke. J Stroke Cerebrovasc Dis 2012; 21: 205-9.

9) Kaneko T, Wada H, Onishi K, et al. Cutoff values of D-dimer and FDP in plasma for the diagnosis of thrombosis. Vasc Dis Prev 2008; 5: 81-8.

10) Nomura H, Wada H, Mizuno T, et al. Negative predictive value of $\mathrm{D}$-dimer for diagnosis of venous thromboembolism. Int J Hematol 2008; 87: 250-5.

11) Wada H, Kobayashi T, Abe Y, et al. Elevated levels of soluble fibrin or D-dimer indicate high risk of thrombosis. J Thromb Haemost 2006; 4: 1253-8.

12) Ota $S$, Wada $H$, Nobori $T$, et al. Diagnosis of deep vein thrombosis by plasma-soluble fibrin or D-dimer. Am J Hematol 2005; 79: 274-80.

13) Editorial committee on Japanese guideline for prevention of venous thromboembolism. Japanese guideline for prevention of venous thromboembolism. Tokyo: Medical Front Int Ltd. 2004; pp. 1-96. (in Japanese)

14) Ota S, Yamada N, Tsuji A, et al. Incidence and clinical predictors of deep vein thrombosis in patients hospitalized with heart failure in Japan. Circ J 2009; 73: 1513-7.

15) Matsuo H, Matsumura M, Nakajima Y, et al. Frequency of deep vein thrombosis among hospitalized non-surgical Japanese patients with congestive heart failure. J Cardiol 2014; 64: 430-4.

16) Kawase K, Okazaki S, Toyoda K, et al. Sex difference in the prevalence of deep-vein thrombosis in Japanese patients with acute intracerebral hemorrhage. Cerebrovasc Dis 2009; 27: 313-9.

17) Ohmori $\mathrm{H}$, Ochi F, Tanuma $\mathrm{N}$, et al. Deep vein thrombosis in patients with severe motor and intellectual disabilities. Ann Vasc Dis 2013; 6: 694-701.

18) Ohmori H, Kanaoka Y, Murata Y, et al. Deep vein thrombosis in patients with severe motor and intellectual disabilities, especially diagnosis and prevention of recurrence for chronic thrombosis-serial changes of sonography and D-dimer. Ann Vasc Dis 2015; 8: 290-6.
19) Prisco D, Grifoni E. The role of D-dimer testing in patients with suspected venous thromboembolism. Semin Thromb Hemost 2009; 35: 50-9.

20) Tanaka S, Nishigami K, Taniguchi N, et al. Criteria for ultrasound diagnosis of deep venous thrombosis of lower extremities. J Med Ultrason 2008; 35: 33-6.

21) Fujita $S$, Hirota $S$, Oda $T$, et al. Deep venous thrombosis after total hip or total knee arthroplasty in patients in Japan. Clin Orthop Relat Res 2000; 375:168-74.

22) Kahn SR, Lim W, Dunn AS, et al. Prevention of VTE in nonsurgical patients: antithrombotic therapy and prevention of thrombosis, 9th ed: American College of Chest Physicians Evidence-Based Clinical Practice Guidelines. Chest 2012; 141: e195S-226S.

23) Yamada N, Hanzawa K, Ota S, et al. Occurrence of deep vein thrombosis among hospitalized non-surgical Japanese patients. Ann Vasc Dis 2015; 8: 203-9.

24) Ministry of Health, Labour and Welfare. Patient survey in 2011; Table 63. Available at http://www.e-stat.go.jp/ SG1/estat/List.do?lid=000001103073. Accessed 6 August, 2015. (in Japanese)

25) Watson HG, Chee YL. Aspirin and other antiplatelet drugs in the prevention of venous thromboembolism. Blood Rev 2008; 22: 107-16.

26) Urayama H, Fukui D, Tanaka K. Diagnostic evaluation of duplex ultrasound for deep vein thrombosis. Jpn J Phlebol 2003; 14: 209-13. (in Japanese)

27) Pedersen OM, Aslaksen A, Vik-Mo H, et al. Compression ultrasonography in hospitalized patients with suspected deep venous thrombosis. Arch Intern Med 1991; 151: 2217-20.

28) Ohgi S, Ohgi N. Relationship between specific distributions of isolated soleal vein thrombosis and risk factors. Ann Vasc Dis 2014; 7: 246-55.

29) Bates SM, Jaeschke R, Stevens SM, et al. Diagnosis of DVT: antithrombotic therapy and prevention of thrombosis, 9th ed: American College of Chest Physicians Evidence-Based Clinical Practice Guidelines. Chest 2012; 141: e351S-418S.

30) Satokawa H, Yamaki T, Iwata H, et al. The survey of deep vein thrombosis and venous thromboembolism prevention: Japanese Vein Study XIII. Jpn J Phlebol 2012; 23: 271-81. (in Japanese) 